\title{
Frugivoria e Dispersão de Sementes de Euterpe edulis Mart. (Arecacea) por Mamíferos e Aves Silvestres na Mata Atlântica do Sul do Brasil
}

\author{
Alexandre Rodrigues da Silva ${ }^{1,2^{*}}$, Rômulo da Rosa Silveira ${ }^{1}$, Augusto Aumond ${ }^{1}$, Anelise Beneduzi da \\ Silveira ${ }^{2} \&$ Cristina Vargas Cademartori ${ }^{1,2}$
}

${ }^{1}$ Laboratório de Conservação e Manejo da Biodiversidade - LabCMBio, Curso de Ciências Biológicas, Universidade La Salle - Unilasalle, Canoas, Rio Grande do Sul, Brasil.E-mails: *alebiologia_rs@hotmail.com, romulor.silveira@gmail.com, augustoaumond@hotmail.com.

${ }^{2}$ Mestrado em Avaliação de Impactos Ambientais, Unilasalle, Canoas, Rio Grande do Sul, Brasil. E-mails: anelise. silveira@unilasalle.edu.br, cristina.cademartori@unilasalle.edu.br.

Abstract. Frugivory and seed dispersal of Euterpe edulis Mart. (Arecaceae) by wild mammals and birds in the Atlantic Forest in southern Brazil. Euterpe edulis Martius (Arecaceae) is considered one of the most important palm trees for the dispersing and pollinating fauna in the Atlantic Forest. However, the predatory exploitation of the palm heart has been causing its decline and extinction throughout the Atlantic Forest. The aim of this study was to identify the interactions between mammals and birds with Juçara palm, as well as contribute to the understanding of the ecological processes of seed dispersal and germination. The study was carried out in the municipality of Maquiné, state of Rio Grande do Sul, in an area of Dense Ombrophylous Forest belonging to Fundação Estadual de Pesquisa Agropecuária (FEPAGRO). Mammals and birds were recorded with six camera traps, focal (tree-focal) observation and transections. Germination experiments were also performed and differences were tested using Biostat 5.3 software. Twelve months of study resulted in over 1300 videos with a sampling effort of 334 camera days. In 1180 videos, the fauna interacted directly with the fruits of $E$. edulis, totaling 1245 interactions. In 38\% of these, fruits were consumed, which were carried in $37 \%$ of these records and in 25\%, swallowed. In total, 17 species were recorded, 12 birds and five mammals. Of these, small rodents were the ones that consumed or carried the fruits the most ( $N=616$ or $65 \%)$. Of the birds, four Turdus species consumed, swallowed or carried 322 fruits (33\%). The percentage and / or the germination speed were significantly higher in regurgitated or pulped seeds by the fauna than in seeds extracted directly from the plant. Large frugivores were eventually recorded, suggesting a defaunation in the study area. The number of interactions verified, however, confirms the ecological importance of $E$. edulis for the maintenance of the frugivorous guild with occurrence in the locality.

Keywords: Juçara palm tree, animal-plant interactions, fruit removal.

Resumo. Euterpe edulis Martius (Arecaceae) é considerada uma das palmeiras mais importantes para a fauna dispersora e polinizadora na Mata Atlântica. Contudo, a exploração predatória do palmito vem causando seu 
declínio e extinção ao longo da Mata Atlântica. O objetivo deste estudo foi identificar as interações entre mamíferos e aves com E. edulis Mart., bem como contribuir para a compreensão dos processos ecológicos de dispersão e germinação de sementes. O estudo foi realizado no município de Maquiné, RS, em área de Floresta Ombrófila Densa pertencente à Fundação Estadual de Pesquisa Agropecuária (FEPAGRO). Mamíferos e aves foram registrados por meio da utilização de seis armadilhas de filmagem, observação focal (árvorefocal) e transecções. Experimentos de germinação foram também realizados e as diferenças encontradas foram testadas por meio do programa Biostat 5.3. Doze meses de estudo resultaram em mais de 1300 vídeos com um esforço amostral de 334 armadilhas-dia. Em 1180 vídeos, a fauna interagiu diretamente com os frutos de E. edulis, totalizando 1245 interações. Em 38\% destas houve consumo dos frutos, os quais foram carregados em $37 \%$ desses registros e em 25\%, engolidos. No total, foram registradas 17 espécies, 12 de aves e cinco de mamíferos. Destes, os pequenos roedores foram os que mais consumiram ou carregaram os frutos ( $N=616$ ou 65\%). Das aves, quatro espécies de Turdus consumiram, engoliram ou carregaram 322 frutos (33\%). 0 percentual e/ou a velocidade de germinação foram significativamente maiores em sementes regurgitadas ou despolpadas pela fauna do que em sementes extraídas diretamente da planta. Grandes frugívoros foram eventualmente registrados, sugerindo um quadro de defaunação na área de estudo. $O$ número de interações constatadas, contudo, confirma a importância ecológica de E. edulis para a manutenção da guilda de frugívoros com ocorrência na localidade.

Palavras-chave: Palmeira-juçara, interações animal-planta, remoção de frutos.

\section{INTRODUÇÃO}

O papel das interações animal-planta, entre elas a dispersão e a predação de sementes, tem ganhado prioridade em programas de conservação (DEL-CLARO \& TOREZAN-SILINGARDI, 2006), pela sua importância na manutenção da integridade das comunidades naturais e, por consequência, aos inúmeros benefícios à humanidade. A análise destas interações, sob o ponto de vista da conservação, auxilia na compreensão dos mecanismos geradores e mantenedores da diversidade biológica, permitindo ações de manejo e recuperação de áreas degradadas, bem como a definição de metodologias de intervenção mais eficientes.
A dispersão de sementes, considerada uma das fases mais críticas do ciclo de vida das plantas (WANG \& SMITH, 2002), é um processo essencial à manutenção da maioria das espécies vegetais no seu habitat natural, sendo a estratégia zoocórica muitas vezes predominante, especialmente em ambientes tropicais e subtropicais (BUDKE et al., 2005; JORDANO et al., 2006). Uma planta zoocórica é capaz de atrair espécies de distintos grupos faunísticos, sendo as aves e os mamíferos os principais aliados nessa estratégia (ReIS \& KAgeYAMA, 2000). Na Mata Atlântica, existe uma grande quantidade de plantas que produzem frutos carnosos, de modo que $20 \%$ e $50 \%$ das espécies de aves e mamíferos consomem esses frutos em algum período do 
ano (JORDANo et al., 2006). Dentre as espécies vegetais, a família das palmeiras (Arecaceae) figura entre as mais importantes para a fauna, por produzirem uma grande quantidade de flores e de frutos (GALETTI \& Aleixo, 1998; REIS \& KAGEYAMA, 2000), provendo recursos alimentares em abundância para polinizadores e dispersores, tais como insetos, aves e mamíferos (Mantovani, 1998; Galetti \& Aleixo, 1998). Além disso, as palmeiras possuem longos períodos de frutificação, o que as torna excelentes fontes de nutrientes para frugívoros, principalmente em épocas de escassez de outros alimentos, já que, no geral, não existe sincronia de frutificação com outras plantas (REIS, 1995; HENDERSON, 2002).

A abundante produção de frutos e a grande gama de animais que deles se alimentam fazem da palmeira-juçara uma espécie-chave na dinâmica florestal, por atrair, também, a fauna que dispersa sementes de outras plantas, assim como seus predadores (REIS, 1995). Devido à extração predatória do palmito, que é a porção comestível do meristema apical e das folhas jovens enroladas, esta palmeira vem sendo eliminada de boa parte da Mata Atlântica (FARIAS, 2009; Troian, 2009; FAVReto et al., 2010), onde seu estado de conservação é vulnerável, encontrando-se igualmente ameaçada na Argentina e no Paraguai (GAletTI \& Fernandes, 1998). No Rio Grande do Sul, em vista da escassez de matéria-prima, o ciclo de extrativismo do palmito está em fase de declínio (FAVRETo, 2010).

Por outro lado, a palmeira-juçara está também sujeita aos efeitos crescentes do extrativismo de seus frutos, utilizados no estado do Rio Grande do Sul como substitutos do açaí, em geral provenientes de Euterpe precatoria (Martius, 1842) e Euterpe oleracea Martius, 1824 (FARIAS, 2009; TROIAN, 2009 e FAVRETo et al., 2010). Por consequência, a espécie consta na lista regional da flora ameaçada de extinção como "em perigo" (FzBRS, 2015) e está entre as plantas que mais sofrem pressões antrópicas na Mata Atlântica, inclusive em decorrência da exploração por agricultores familiares com baixa renda do litoral norte do estado (COELHo de SouzA, 2003).

A palmeira $E$. edulis pode produzir uma grande quantidade de frutos em áreas preservadas (GALETTI \& ALEIXO, 1998), fornecendo alimento para pelo menos 58 espécies de aves e 21 espécies de mamíferos (GALETTI et al., 2013). A despeito de sua importância ecológica, as palmeiras encontram-se suscetíveis à extinção devido à exploração ilegal do palmito, à perda de hábitats e à defaunação, que comprometem, por exemplo, a presença de grandes frugívoros nos remanescentes florestais (GALETTI \& AleiXo, 1998; PIRES, 2006). Entre as espécies de vertebrados mais afetadas pela fragmentação, estão aves e mamíferos de médio e grande porte, que atuam como importantes dispersores e predadores de sementes de palmeiras (SILVA \& TABARElli, 2001; Cordeiro \& HoWe, 2003; TERBorgh et al., 2008; ANDREAZzı et al., 2009). Esse cenário de devastação e superexploração foi observado dentro e nas proximidades da área de estudo, o que vem provocando o declínio das 
populações de E. edulis na região (RAUPP et al., 2009; FAVRETO, 2010).

A maioria dos estudos referentes à frugivoria e à dispersão dos frutos da palmeirajuçara tem como foco o consumo e as interações por espécies de aves (WILLIS, 1989; ZIMMERMANN, 1999; LAPS, 1996; REIS \& KAGEYAMA, 2000; PIzo et al., 2002; CASTRO, 2003; CôRTES, 2006), sendo escassos os estudos de frugivoria por mamíferos (GALETTI et al., 1999; CAMPos et al., 2012), já que este grupo raramente é observado consumindo os frutos da palmeira (GALETTI et al., 1999). Considerando-se, então, a importância de Euterpe edulis em razão das interações ecológicas que estabelece com várias espécies de aves e mamíferos (WILLIS, 1989; LAPS, 1996; GALETTI et al., 1999; ZIMMERMANN, 1999; REIS \& KAgEYAMA, 2000; PIzo et al., 2002; CASTRO, 2003; GALETTI et al., 2003; CÔRTES, 2006; CAMPOS et al., 2012), buscou-se: (A) identificar as espécies de aves e mamíferos que consomem frutos; (B) quantificar as interações entre mamíferos e aves silvestres com a palmeira-juçara e (C) testar a taxa de germinação pré e pós-dispersão, visando ampliar a compreensão sobre os processos ecológicos de dispersão e germinação das sementes, e contribuir para o manejo e conservação da palmeira ameaçada.

\section{MATERIAL e Métodos}

\section{ÁREA DE ESTUdo}

O estudo foi realizado no município de Maquiné (2940’30"S e 5012'26"W), localizado no Litoral Norte do Rio Grande do Sul, a aproximadamente $12 \mathrm{~m}$ de altitude (DALPIAZ, 2000). A área de estudo faz parte de uma propriedade de 367 ha, localizada na RS 484, próxima ao rio Maquiné, em área de Floresta Ombrófila Densa com ocorrência da palmeira $E$. edulis. Trata-se de uma propriedade da Fundação Estadual de Pesquisa Agropecuária - FEPAGRO (Figura 1). O clima da região, segundo Köppen, é do tipo Cfa ou subtropical úmido, onde a média anual de temperatura é de $19,9{ }^{\circ} \mathrm{C}$ e a pluviométrica, de $1731 \mathrm{~mm}$ bem distribuídos ao longo do ano. A temperatura média do mês mais quente (janeiro) é de $24,5^{\circ} \mathrm{C}$ e do mês mais frio (julho) é de $15,5^{\circ} \mathrm{C}$ (Model \& SANDER, 1999).

A bacia hidrográfica do rio Maquiné, na encosta Atlântica do Litoral Norte gaúcho, foi uma das regiões selecionadas como áreas-piloto da Reserva da Biosfera da Mata Atlântica no Rio Grande do Sul (MARCUZzo, 1998). Segundo BECKER (2002), 93\% (505,5 km²) da bacia do rio Maquiné era coberta por florestas antes da colonização europeia e os 7\% restantes eram cobertos por campos nativos nas localidades acima de 800 $\mathrm{m}$ de altitude. Atualmente, atividades humanas como a pecuária e a agricultura vêm modificando a estrutura original da maior parte da área da bacia e toda a região é afetada por problemas como o extrativismo de espécies nativas (FAVReto, 2010). A cidade de Maquiné possui um microcentro urbano e algumas fazendas distribuídas pelo interior. Habitada por pequenas famílias de agricultores e pescadores, tem como principais atividades econômicas a produção agrícola, de tijolos e telhas (olarias), além da 

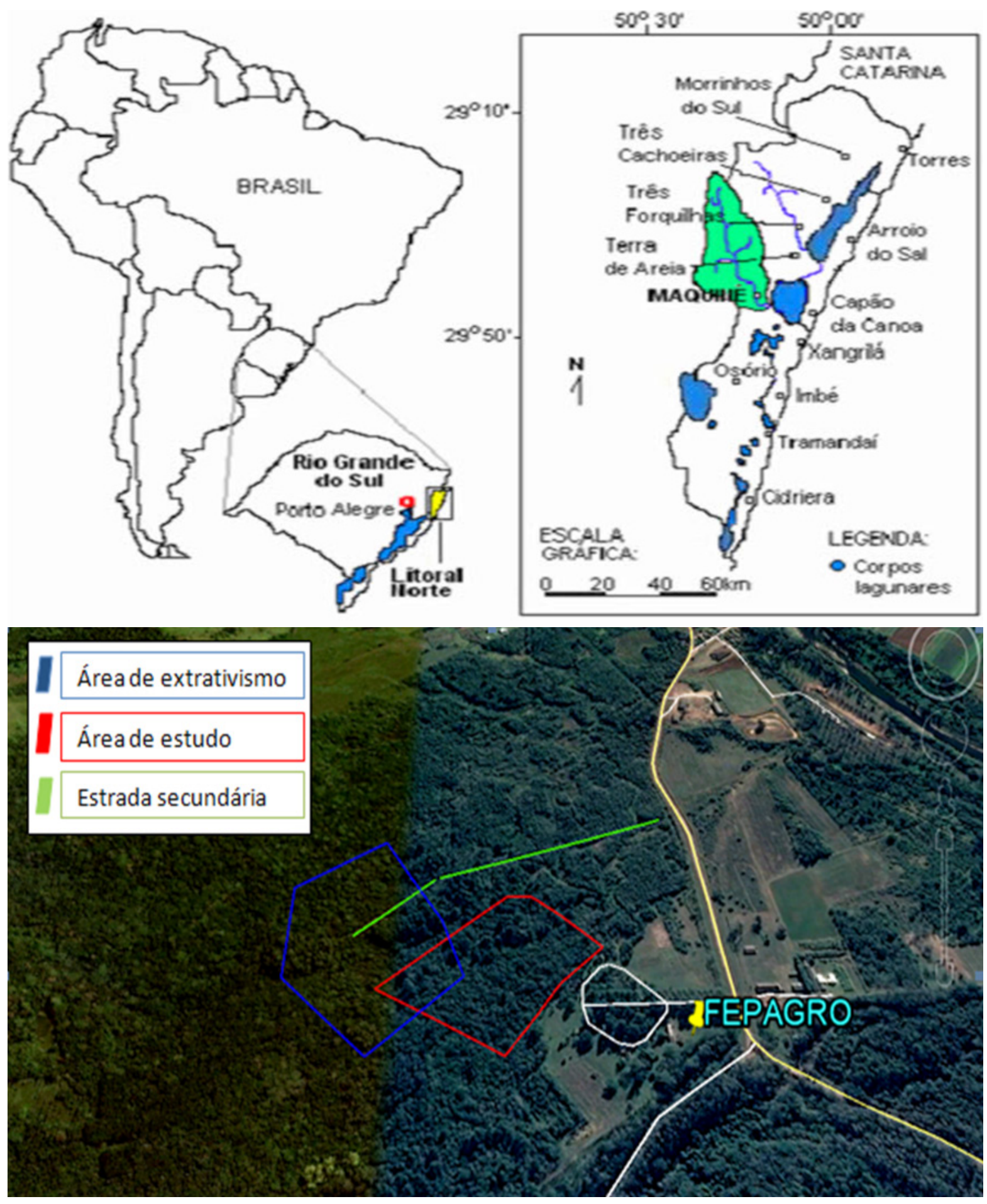

Figura 1. Localização da área de estudo, pertencente à FEPAGRO, município de Maquiné, Rio Grande do Sul. (A) Mapa do Brasil e do Rio Grande do Sul com a localização do município de Maquiné em destaque e (B) Imagem de satélite com a delimitação da área de estudo (Fontes: Maquiné, 2015 \& GoogLE EARTH, 2016). 
pecuária e o extrativismo de produtos vegetais florestais como Rumohra adiantiformis (G. Forst.) ou samambaia-preta, Euterpe edulis (palmito) e plantas epífitas (LEMOS, 2003).

O entorno de Maquiné se caracteriza por apresentarextensasáreas comformações vegetais conectadas com 17 unidades de conservação (FAVReto, 2010). Apesar dos impactos negativos evidenciados na área de estudo ou em suas proximidades, como a extração ilegal de palmito e a presença de animais domésticos (cães e gatos), há uma boa conectividade com outros remanescentes florestais e aproximadamente um hectare preservado com ocorrência de indivíduos adultos de $E$. edulis produzindo frutos. (R. FAVRETo, comunicação pessoal, 2014).

\section{Procedimentos DE CAMPO}

Foram realizadas 12 expedições entre setembro de 2014 a agosto de 2015. As amostragens transcorreram durante três a quatro dias consecutivos ou até uma semana em fases de maior frutificação, totalizando 60 dias de amostragem.

Mamíferos e aves foram registrados por meio da utilização de seis armadilhas fotográficas e de filmagem (Bushnell Trophy Cam XLT) mantidas em funcionamento por 24 horas/dia no decorrer de todo o período de amostragem, totalizando seis unidades amostrais. As armadilhas foram dispostas de duas formas: A) no solo, para monitorar a fauna que se alimentava dos frutos caídos e B) fixadas em galhos de árvores e voltadas para os cachos ou em plataformas suspensas que continham frutos, para monitorar a fauna arborícola. Em épocas em que as palmeiras frutificavam, três armadilhas ficaram direcionadas para o solo e três voltadas para os cachos. Em épocas de redução ou escassez de frutos nos cachos, as armadilhas ficavam direcionadas para o solo com frutos acumulados, coletados em áreas adjacentes. As armadilhas foram programadas para filmagens de 20 segundos a cada movimento registrado. O esforço amostral resultou do produto entre o número de armadilhas efetivamente expostas (armadilhas que porventura apresentaram falhas no mecanismo foram desconsideradas) e o número total de dias de amostragem (no armadilhas $x \mathrm{n}$ ㅇ dias por amostragem), onde cada dia correspondeu a um período de 24 horas, totalizando 334 armadilhas-dia.

A atividade de frugivoria por aves foi avaliada também por observações focais (árvorefocal) e transecções, conforme GALETTI et al. (1999, 2003) e CULLEN JR. \& RUdRAN (2003). O método de observação focal foi realizado nas primeiras horas da manhã dos meses de setembro a novembro de 2014 e abril a agosto de 2015 , totalizando 40 horas de esforço e nove diferentes palmeiras observadas. As observações foram realizadas por meio de binóculo, por até dois observadores a cada amostragem.

A captura de pequenos mamíferos não voadores, utilizando-se armadilhas do tipo Sherman, também foi empregada para confirmação da identificação taxonômica das espécies de pequenos roedores silvestres 
registrados nas filmagens. 0 esforço foi equivalente a 48 armadilhas-noite.

\section{Manipulação de sementes por aves e MAMÍFEROS}

Os comportamentos da fauna em relação aos frutos da palmeira-juçara, identificados nas filmagens, foram agrupados em três categorias: A) despolpadores, quando os indivíduos apenas consumiam a polpa (ou parte dela) e deixavam a semente intacta; B) carregadores, quando os frutos eram manipulados e transportados para longe do campo de filmagem; e C) engolidores, quando os frutos eram engolidos na frente da armadilha de filmagem.

\section{COLETA DE SEMENTES E TESTES DE GERMINAÇÃo}

As sementes manipuladas por roedores foram coletadas após a realização das filmagens, enquanto as sementes manipuladas ou dispersadas por aves foram recolhidas durante as observações visuais. Os testes de germinação foram realizados, então, com sementes obtidas in loco, após sua manipulação pela fauna. Também foram desenvolvidos testes de germinação com grupos-controle (sementes extraídas diretamente da planta), para fins de comparação. Os experimentos foram realizados no laboratório de sementes da FEPAGRO de Porto Alegre, estado do Rio Grande do Sul. As sementes foram dispostas em pequenas caixas plásticas, em substratos de areia esterilizada, e colocadas em estufas, onde permaneceram em temperatura alternada entre $20^{\circ} \mathrm{C}(16 \mathrm{~h})$ e $30^{\circ} \mathrm{C}(8 \mathrm{~h})$, ao longo de quatro meses. Compreenderam cinco grupos- controle e oito grupos-teste. Os experimentos foram acompanhados em intervalos de 15 dias. O surgimento de primórdios radiculares foi considerado evidência de germinação.

\section{ANÁLISE DE DADOS}

O teste de Kruskal-Wallis foi aplicado para avaliar a significância das diferenças encontradas entre os comportamentos de manipulação dos frutos pela fauna (despolpar, carregar ou engolir). Também foi utilizado para avaliar as diferenças entre o número de interações registradas entre os distintos grupos faunísticos.

Os resultados de germinação foram avaliados separadamente por espécie ou grupo faunístico, comparando-se a porcentagem de sementes que germinaram no grupo-teste com aquela do grupo-controle. As diferenças encontradas entre as médias foram avaliadas por meio do Teste t com correção de Welch (que assume variâncias distintas entre as amostras).

O Teste Exato de Fisher foi utilizado para amostras pequenas, comparando-se a frequência de sementes que germinaram no grupo-teste em relação ao grupo-controle. A normalidade dos dados foi avaliada por meio do Teste de Kolmogorov-Smirnov. As análises foram realizadas por meio do programa Biostat 5.3 (AYRES et al., 2007). As diferenças foram consideradas a um nível de significância equivalente ou inferior a $5 \%$.

O índice de velocidade de germinação (IVG), em que o número de sementes ou plântulas 
é observado e contabilizado, foi obtido de acordo com BORGHETTI \& FERREIRA (2004), pela equação: IVG $=\mathrm{G} 1 / \mathrm{N} 1+\mathrm{G} 2 / \mathrm{N} 2+\ldots \mathrm{Gn} / \mathrm{Nn}$, onde $\mathrm{G}$ representa número de plântulas computadas nas contagens e $\mathrm{N}$ número de dias após a montagem do experimento.

\section{RESULTADOS}

Ao longo de um ano foram registradas 17 espécies de mamíferos e aves interagindo com a palmeira Euterpe edulis. Mais de 1300 vídeos foram analisados, em 1180 dos quais a fauna interagiu diretamente com os frutos de E. edulis, totalizando 1245 interações. Em 471 destas interações, os frutos foram manipulados e a polpa consumida sem que a semente fosse engolida, enquanto que em 465 os frutos foram carregados e em 309, engolidos inteiros. No total das filmagens foram registradas 12 espécies de animais, sendo cinco mamíferos e sete aves (Tabela 1). Mamíferos interagiram 807 vezes com o fruto da palmeira, enquanto aves interagiram 394 vezes.

Os distintos comportamentos da fauna em relação aos frutos diferiram significativamente entre si $(H=15,3568 ; p=0,0006)$. Dentre as categorias, "despolpar" e "carregar" diferiram significativamente de "engolir". Os pequenos roedores, Oligoryzomys nigripes (Olfers, 1818) e Sooretamys angouya (G. Fischer, 1814), foram os principais responsáveis por essa diferença (Tabela 1). Ao se comparar os grupos faunísticos (O. nigripes, S. angouya, Turdus spp., Penelope

Tabela 1. Comportamentos de interação de espécies de mamíferos e aves com os frutos de Euterpe edulis em área de Floreta Ombrófila Densa, em Maquiné, RS, registrados através de armadilhas fotográficas.

\begin{tabular}{|c|c|c|c|c|c|c|c|}
\hline \multirow[b]{2}{*}{ Classe } & \multirow[b]{2}{*}{ Espécie } & \multirow[b]{2}{*}{ Nome popular } & \multirow{2}{*}{ 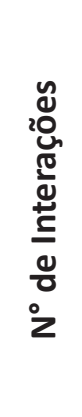 } & \multirow{2}{*}{ 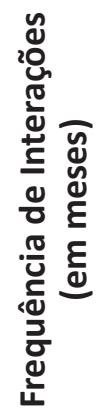 } & \multicolumn{3}{|c|}{ Comportamento } \\
\hline & & & & & 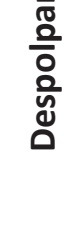 & 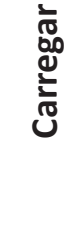 & 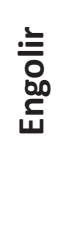 \\
\hline \multirow{5}{*}{ Mammalia } & Didelphis albiventris Lund, 1840 & $\begin{array}{l}\text { Gambá-de-orelha- } \\
\text { branca }\end{array}$ & 2 & 2 & 2 & 0 & 0 \\
\hline & $\begin{array}{c}\text { Dasypus novemcinctus Linnaeus, } \\
1758\end{array}$ & Tatu-galinha & 4 & 3 & 4 & 0 & 0 \\
\hline & $\begin{array}{c}\text { Cerdocyon thous (Linnaeus, } \\
\text { 1758) }\end{array}$ & Graxaim & 2 & 1 & 2 & 0 & 0 \\
\hline & $\begin{array}{c}\text { Oligoryzomys nigripes } \\
1818 \text { ) }\end{array}$ & Rato-do-arroz & 367 & 9 & 133 & 234 & 0 \\
\hline & $\begin{array}{c}\text { Sooretamys angouya (G. Fischer, } \\
\text { 1814) }\end{array}$ & Rato-do-arroz & 432 & 11 & 218 & 214 & 0 \\
\hline
\end{tabular}


Tabela 1. Continução.

\begin{tabular}{|c|c|c|c|c|c|c|c|}
\hline & $\begin{array}{c}\text { Crypturellus tataupa (Temminck, } \\
\text { 1815) }\end{array}$ & Inhambu-chintã & 3 & 1 & 3 & 0 & 0 \\
\hline & Penelope sp. & Jacu & 17 & 2 & 3 & 0 & 14 \\
\hline & Leptotila sp. & Pomba-do-mato & 2 & 2 & 2 & 0 & 0 \\
\hline \multirow[t]{4}{*}{ Aves } & $\begin{array}{l}\text { Chamaeza campanisona } \\
\text { (Lichtenstein, 1823) }\end{array}$ & Tovaca-campainha & 4 & 1 & 4 & 0 & 0 \\
\hline & Turdus rufiventris Vieillot, 1818 & Sabiá-laranjeira & 38 & 5 & 22 & 16 & 0 \\
\hline & Turdus albicollis Vieillot, 1818 & Sabiá-coleira & 322 & 5 & 13 & 15 & 294 \\
\hline & Turdus leucomelas Vieillot, 1818 & Sabiá-barranco & 52 & 4 & 45 & 6 & 1 \\
\hline
\end{tabular}

sp. e outros) quanto ao número de interações com os frutos, observou-se diferença significativa $(H=15,3568 ; p=0,004)$ apenas entre Penelope sp. e S. angouya, cujo número de interações e sua frequência, em meses, foi muito superior (Tabela 1).

\section{PADRÕES DE FRUGIVORIA POR MAMÍFEROS}

Dentre os mamíferos, os pequenos roedores apresentaram o maior número de interações, representados por duas espécies: Sooretamys angouya e Oligoryzomys nigripes
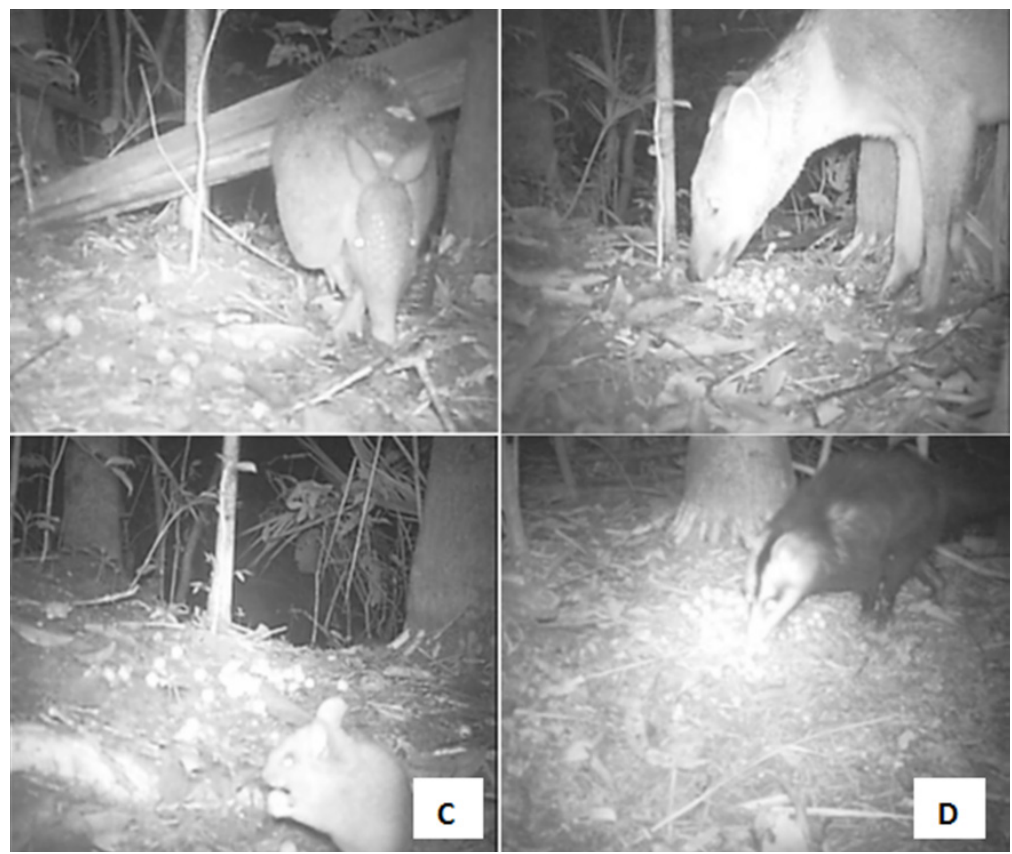

Figura 2. Mamíferos que interagiram com os frutos de Euterpe edulis em área de Floresta Ombrófila Densa, em Maquiné, RS, registrados por filmagem. (a). Dasypus novemcinctus (Tatu-galinha); (b). Cerdocyon thous (Graxaim-do-mato); (c). Sooretamys angouya (Rato-do-arroz) e (d). Didelphis albiventris (Gambá-de-orelha-branca). 
(Tabela 1). Juntas, estas duas espécies foram as que mais despolparam ou carregaram os frutos ( $N=799$ ou 64\%). Também foram registradas outras três espécies de mamíferos, Didelphis albiventris Lund, 1840, Dasypus novemcinctus Linnaeus, 1758 e Cerdocyon thous (Linnaeus, 1758) (Figura 2). D. novemcinctus e C. thous interagiram com os frutos diretamente no solo, enquanto $D$. albiventris interagiu também no estrato arbóreo (a $6 \mathrm{~m}$ de altura). O consumo de frutos por $C$. thous também foi evidenciado de forma indireta, por conteúdo fecal, no qual foram encontradas dez sementes de $E$. edulis intactas.

\section{PADRÕES DE FRUGIVORIA POR AVES}

Todas as espécies de aves foram observadas consumindo os frutos diretamente no solo (Figura 3). O gênero Turdus, representado por três espécies nas filmagens, T. albicollis Vieillot, 1818, T. leucomelas Vieillot, 1818 e T. rufiventris Vieillot, 1818, despolpou, engoliu ou carregou um total de 412 frutos (34\%). Dentre as aves, $T$. albicollis foi a que mais interagiu com os frutos da palmeira, principalmente engolindo os frutos inteiros (Tabela 1). Turdus leucomelas e $T$. rufiventris, por sua vez, predominantemente carregaram ou despolparam frutos depositados

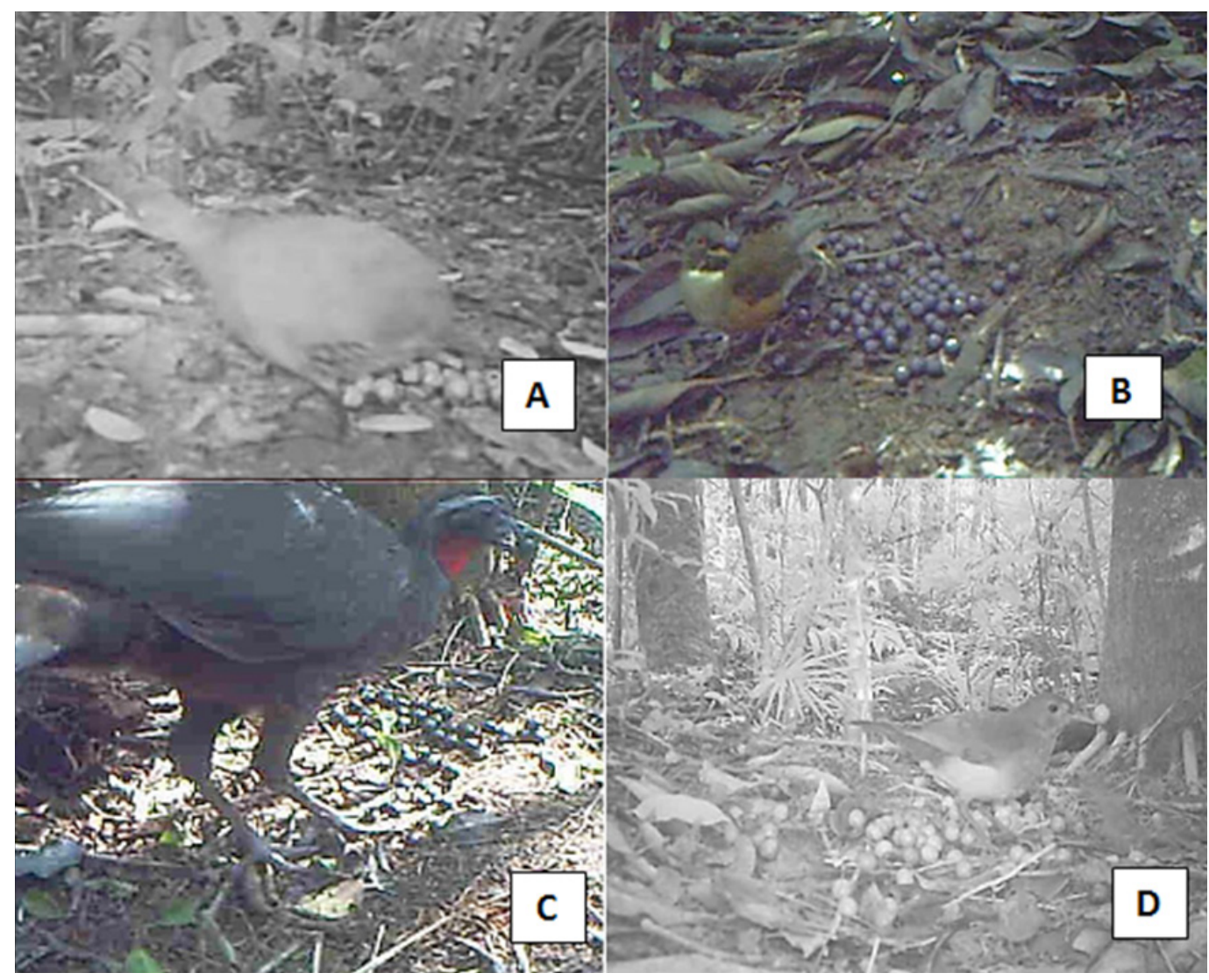

Figura 3. Aves que interagiram com os frutos de Euterpe edulis em área de Floresta Ombrófila Densa, em Maquiné, RS, registradas por filmagens. (a). Crypturellus tataupa (Inhanbu-chintã); (b). Turdus albicollis (Sabiá-coleira); (c). Penelope sp. (Jacu) e (d). Turdus leucomelas (Sabiá-barranco). 
no solo (Tabela 1). Houve apenas um registro de T. leucomelas engolindo um fruto inteiro.

Penelope sp. obteve o quarto maior número de interações com os frutos, apenas em duas amostragens, setembro de 2014 e agosto de 2015 (Tabela 1). Por sua vez, os registros de Crypturellus tataupa (Temminck, 1815), Leptotila sp. e Chamaeza campanisona (Lichtenstein, 1823) somaram nove interações (Tabela 1). O comportamento dessas três espécies em relação aos frutos foi de bicá-los sem retirar totalmente a polpa.

Mais cinco espécies de aves foram registradas pelos métodos de árvore-focal e transecção, Pyrrhura frontalis (Vieillot, 1817), Thalurania glaucopis (Gmelin, 1788), Trogon surrucura Vieillot, 1817, Turdus amaurochalinus (Cabanis, 1850) e Ramphastos dicolorus Linnaeus, 1766, totalizando 12 espécies em interação com a palmeira. Duas foram visualizadas regurgitando sementes, T. albicollis e $R$. dicolorus. Os frutos foram consumidos tanto por espécies que visitaram os cachos em frutificação ( $T$. albicollis e $P$. frontalis) quanto por espécies que coletaram os frutos preferencialmente em voo (T. surrucura). Bandos de três a seis indivíduos de $P$. frontalis foram vistos despolpando os frutos da palmeira, assim como T. rufiventris, que apenas bicou o fruto no solo para retirar a polpa sem engolir a semente. Registros de sementes sendo regurgitadas por $T$. albicollis foram obtidos em três oportunidades. T. glaucopis visitou o cacho apenas. T. amaurochalinus, por sua vez, foi avistado carregando um fruto retirado diretamente do cacho.

\section{GERMINAÇÃo DE SEMENTES}

Os testes de germinação, realizados após o consumo dos frutos de E. edulis pela fauna, demonstraram que as diferenças encontradas não se deveram ao acaso. Considerando-se a amostra de sementes cujos frutos foram consumidos por roedores, houve diferença significativa entre o percentual médio de germinação do grupoteste, equivalente a $77,97 \%$, e a média do grupocontrole, que foi inferior e igual a $14,67 \%$ ( $t=$ 6,$846 ; g l=7 ; p=0,0002$ ). A velocidade média de germinação também diferiu significativamente entre os dois grupos, tendo sido maior, igualmente, no grupo-teste $(\mathrm{t}=6,707 ; \mathrm{gl}=4 ; \mathrm{p}$ $=0,0026)$.

Houve diferença significativa no percentual médio de germinação do grupo-teste proveniente de sabiás $(76,52 \%)$ e a média do grupo-controle (20\%) ( $t=4,279 ; g l=3 ; p=0,0234)$, assim como na velocidade de germinação, que foi 16 vezes mais rápida no grupo-teste $(t=4,530$; $g \mid=2 ; p=0,0454)$. Também houve diferença significativa ( $p=0,0002$ ) entre o percentual de germinação do grupo-teste de uma amostra de $P$. frontalis ( $P G=39 \%$ ) e o do grupo-controle (PG = $3 \%)$. A velocidade de germinação nessa amostra foi 40 vezes maior no grupo-teste (IVG $=0,40$ ) do que no controle (IVG $=0,01$ ). Em uma segunda amostra referente a esta mesma espécie, o percentual de germinação do grupo-teste (PG $=41 \%)$ também diferiu significativamente $(p$ $=0,0118)$ do grupo-controle $(P G=13 \%)$ e a 
velocidade de germinação foi nove vezes maior no grupo-teste (IVG $=0,63$ ) do que no controle (IVG $=0,07$ ). A amostra de sementes ingeridas por C. thous não apresentou diferença significativa $(p=0,1206)$ nos percentuais de germinação do grupo-teste $(P G=16 \%)$ em relação ao grupocontrole (IVG $=4 \%$ ).

\section{DISCUSSÃo}

Os resultados deste estudo demonstraram elevadas taxas de consumo dos frutos de $E$. edulis por espécies de pequeno porte $(<1 \mathrm{~kg})$ em $97,5 \%$ das filmagens e $43 \%$ dos avistamentos de aves. Aves de médio porte totalizaram quase $50 \%$ dos avistamentos, enquanto que as de grande porte apenas 7,5\%. Em apenas 2,5\% das filmagens houve registro de aves e mamíferos de médio e grande porte ( $>1 \mathrm{~kg}$ ). Este cenário difere da maioria dos estudos realizados na Mata Atlântica, que demonstram uma maior interação da palmeira com espécies animais de maior porte (LAPS, 1996; GALETTI et al., 1999; ZIMMERMANN, 1999; ReIS \& Kageyama, 2000; Campos et al., 2012). Isso pode ser explicado pelo fato de que as áreas amostradas em outros estudos são mais preservadas e não estão submetidas aos inúmeros e intensos impactos que caracterizam o litoral norte do Rio Grande do Sul (FAVReto, 2010).

O consumo parcial ou total do epicarpo e do mesocarpo dos frutos (polpa), principalmente por roedores e espécies do gênero Turdus, foi relativamente elevado ( $38 \%$ das filmagens), distinguindo-se os indivíduos que carregavam os frutos (37\%) dos que os engoliam (25\%). Turdus albicollis e Penelope $\mathrm{sp}$. foram os principais táxons a engolir os frutos inteiros, o que também foi constatado em outros estudos sobre dispersão de E. edulis (MIKICH 2002; FADINI, 2005; GaletTI, 1999) . Crypturellus tataupa, Chamaeza campanisona e Leptotila sp., por sua vez, consumiram parcialmente a polpa dos frutos que se encontravam no solo. WILLIS (1979) considera C. tataupa e Leptotila sp., aves granívoras, enquanto alguns estudos citam $C$. tataupa como predadora das sementes de E. edulis (LABECCA, 2012; RIBEIRO, 2012).

Sabiás e roedores aumentaram e aceleraram a germinação das sementes de $E$. edulis nos testes realizados. As espécies de aves do gênero Turdus, assim como os pequenos roedores, foram os grupos que mais interagiram com os frutos da palmeira na área de estudo. O transporte de frutos foi realizado por $T$. rufiventris, T. albicollis e T. leucomelas em 37 (3\%) oportunidades, mas o comportamento de engolir os frutos foi característico de T. albicollis. Turdus albicollis e T. amaurochalinus, ainda, foram as duas espécies observadas carregando os frutos retirados diretamente dos cachos. As espécies deste gênero apresentam diferentes hábitos e técnicas de captura de frutos (FADINI, 2005; CORTÊs, 2006), sendo considerados os principais dispersores de E. edulis (ZIMMERMANN, 1990). São importantes, ainda, pelo hábito onívoro e por frequentarem ambientes diversos (florestas preservadas, áreas de campo e até mesmo urbanas), fazendo com que, na maioria 
das vezes, as sementes cheguem pela primeira vez nessas áreas após serem regurgitadas (REIS, 1995). Turdus albicollis foi a espécie mais importante quanto ao consumo e dispersão de sementes de $E$. edulis, pelo maior número de interações com os frutos, por ter sido a espécie que mais engoliu e, consequentemente, regurgitou sementes, além de ter sido registrada por meio de todas as metodologias empregadas. A interação de T. albicollis com E. edulis também foi constatada por outros autores (LAPS, 1996; MATOS \& WATKINSON, 1998; ZIMMERMANN, 1999; CASTRO, 2003; CÔRTES, 2006; VON MATTER, 2008; GALETTI et al., 2009; CAMPOs et al. 2012), vários dos quais a identificam como uma das principais espécies a consumir frutos da palmeira-juçara.

Pyrrhura frontalis foi responsável por manipular e despolpar muitos frutos junto à planta-matriz, aspecto também já descrito na literatura (LAPS, 1996; GALLETTI, 1999; REIS \& KAgEyAmA, 2000; CAStRo, 2003; BARRoso, 2010), o que lhe confere uma efetividade baixa de dispersão. A boa capacidade de manipulação faz dos psitacídeos excelentes despolpadores (SICK, 2001). Muitos autores, porém, relacionam o comportamento de $P$. frontalis com a predação de sementes de E. edulis (LAPS, 1996; LABECCA, 2012; RiBEIRO, 2012). Os dados desta pesquisa, contudo, assim como os de REIS (1995), evidenciam a família Psittacidae como derrubadores e despolpadores arborícolas, e não predadores. As sementes despolpadas por P. frontalis também apresentaram percentual e velocidade de germinação superiores, reforçando sua importância como potencial espécie dispersora.

Crypturellus tataupa, Penelope sp. e Ramphastos dicolorus foram as maiores aves registradas. Penelope sp., inclusive, foi a segunda espécie que mais engoliu frutos. As famílias dos Cracídeos e dos Ramphastídeos geralmente estão presentes em listas de espécies que consomem os frutos e dispersam as sementes de E. edulis (GALETTI et al., 1999; Reis \& KAgEYAMA, 2000; FADINI, 2005; CôRTES, 2006; TROIAN, 2009). MIKICH (2002), em estudo realizado no centrooeste do Paraná, na Mata Atlântica, sobre a dieta de Penelope superciliaris, observou que $82 \%$ das amostras fecais analisadas continham sementes de $E$. edulis, apontando os frutos da palmeira como essenciais a sua sobrevivência na região. Aves de grande porte, como os tucanos e os jacus, são considerados dispersores efetivos de $E$. edulis, devido a sua capacidade de engolir e dispersar a semente longe da planta-matriz (GALETTI et al., 2013). Outros autores referemse a $R$. dicolorus como uma ave regurgitadora de sementes da palmeira que pode explorar áreas favoráveis para germinação (GALETTI et al., 1999; FADINI, 2005; CôRTES, 2006).

A família dos Trogonídeos, representada nesta pesquisa por Trogon surrucura, compreende espécies frequentes em estudos de frugivoria e dispersão de sementes de E. edulis (LAPS, 1996; GALETTI et al., 1999; CASTRO, 2003; CORTÊs, 2006). Esta espécie é considerada um grande frugívoro por possuir largura do bico maior que $18 \mathrm{~mm}$ (RIBEIRo, 2012). Apesar de duas espécies deste 
gênero terem sido avistadas na área, apenas $T$. surrucura foi visualizada interagindo diretamente com os frutos da palmeira.

Euterpe edulis pode produzir uma grande quantidade de frutos em áreas preservadas (GAlETTI \& AleiXo, 1998), fornecendo alimento para pelo menos 58 espécies de aves e 21 espécies de mamíferos (GALETTI et al., 2013). GALETTI et al. (1999) estudaram as interações de aves e mamíferos com os frutos da palmeira no sudeste do Brasil e, ao compilarem resultados obtidos por LAPS (1996) na mesma área, reuniram 18 gêneros de aves e 10 de mamíferos, totalizando 32 espécies, 21 de aves $(65,6 \%)$ e 11 de mamíferos (34,4\%). Segundo os autores, em áreas de Mata Atlântica mais preservadas, até 15 espécies de mamíferos (incluindo roedores e morcegos) e 25 de aves poderiam ser registradas consumindo os frutos de E. edulis. Em Florianópolis, SC, CAMPos et al. (2012) constataram sete espécies, sendo três aves (dentre as quais T. albicollis e uma espécie da família Ramphastidae) e quatro mamíferos (dentre os quais duas espécies de pequenos roedores da família Cricetidae). Apenas 12 espécies de aves e cinco espécies de mamíferos foram registradas interagindo com os frutos da palmeira na área de estudo, embora outras espécies frugívoras potencialmente dispersoras tenham sido avistadas próximas das palmeiras em fase de frutificação e maturação dos frutos.

Dentre os mamíferos de médio e grande porte registrados , $D$. novemcinctus foi filmado se alimentando dos frutos da palmeira-juçara em algumas oportunidades. Alguns autores relatam o consumo de frutos de outras palmeiras por esta espécie, como Syagrus romanzoffiana (ANDREAZZI et al., 2009) e Attalea humilis (ANDREAZZI, 2008). REDFORD (1985) afirma que espécies do gênero Dasypus são insetívoras generalistas que podem procurar outros recursos em épocas de escassez de alimento. Didelphis albiventris, por sua vez, foi registrada em poucas oportunidades e, assim como para $D$. novemcinctus, não existem, na literatura, evidências de consumo dos frutos pela espécie. BARRoso et al. (2010) citam, para SP, a partir de relatos de entrevistas em comunidades quilombolas, que Didelphis aurita consome os frutos da palmeira-juçara. Cerdocyon thous, registrada através das filmagens e pelo conteúdo fecal, que continha sementes de E. edulis, também é mencionada por GALLETI et al. (1999). $\mathrm{O}$ baixo registro de espécies de médio e grande porte, neste estudo, pode estar relacionado aos impactos antrópicos registrados em toda a região. Essa defaunação implica em consequências negativas à dispersão de E. edulis, já que apenas quatro aves e um mamífero são considerados capazes de dispersar as sementes em longas distâncias.

GALETTI et al. (2015) registraram 1228 eventos de animais que visitaram E. edulis na região sudeste do Brasil, em SP, compreendendo 14 espécies em área não defaunada (incluindo $C$. tataupa e três espécies de pequenos roedores) e 12 em área defaunada (entre as quais Oligoryzomys sp. e Akodon sp.). Os pequenos roedores apresentaram o maior número de 
registros, assim como neste estudo, mas foram apontados como predadores de sementes (envolvidos em 98,3\% dos casos de predação na área defaunada e 63,5\% na área não defaunada). Todavia, assim como em CAMPos et al. (2012), os pequenos roedores registrados neste estudo evidenciaram apenas o comportamento de consumo total da polpa dos frutos, sem danificar as sementes. REIS (1995) também classificou os pequenos roedores como despolpadores de frutos próximos à planta-matriz e os enquadrou na categoria de dispersores secundários que eventualmente podem transportaros propágulos. $\mathrm{O}$ transporte de frutos pelos pequenos roedores representou $36 \%$ das interações registradas durante este estudo e os frutos foram levados tanto para longe da planta-matriz quanto para o interior de tocas próximas (provavelmente para estoque), e até mesmo para o estrato arbóreo, fato observado principalmente para $O$. nigripes (espécie escansorial, segundo CADEMARTORI et al., 2008). Embora alguns trabalhos relatem a predação de sementes de $E$. edulis por pequenos roedores (PIZO \& SIMÃO, 2001; VIEIRA et al., 2003; PIZO \& VIEIRA, 2004; PINTO et al., 2009), os comportamentos observados, associados aos resultados de germinação (tanto a velocidade quanto a porcentagem de germinação foram significativamente maiores no grupo-teste), revelaram que as duas espécies de pequenos roedores aqui registradas podem dispersar as sementes de E. edulis. Apesar de não ter sido possível conhecer o destino final das sementes transportadas pelos roedores, é provável que alguns dos propágulos tenham sido dispersados secundariamente por eles (FORGET, 1990; VANDER WALL, 1994; BREWER \& REJMÁNEK, 1999), uma vez que em florestas tropicais grande parte das famílias de plantas tem suas sementes dispersas por roedores (VAN RoOSMALEN, 1985), incluindo as palmeiras (TABARELLI \& MANTOVANI, 1999).

A despeito de sua importância ecológica, as palmeiras se encontram suscetíveis à extinção devido à exploração ilegal do palmito, à perda de hábitats e à defaunação, que comprometem, por exemplo, a presença de grandes frugívoros nos remanescentes florestais (GALETTI \& ALEIXO, 1998; PIRES, 2006). Entre as espécies de vertebrados mais afetadas pela fragmentação estão aves e mamíferos de médio e grande porte, que atuam como importantes dispersores e predadores de sementes de palmeiras (SILVA \& TABARELLI, 2001; CORDEIRO \& HOWE, 2003; TERBORGH et al., 2008; ANDREAzZI et al., 2009). Neste estudo, ao longo de um ano, foram registradas apenas 17 espécies de aves e mamíferos de médio e grande porte, corroborando esse cenário de superexploração e defaunação, que vem provocando o declínio das populações de E. edulis na região (RAUPP et al., 2009; FAVRETO, 2010).

\section{CONCLUSÕES}

Os pequenos roedores, assim como as espécies de aves do gênero Turdus, estão bastante associados com os frutos de Euterpe edulis na área de estudo e são capazes de atuar como dispersores das sementes desta palmeira.

Turdus albicollis revelou-se como a mais importante espécie em relação ao consumo e 
dispersão das sementes de $E$. edulis. Indivíduos desta espécie de sabiá são importantes dispersores em bordas florestais e áreas alteradas pela ação antrópica.

A exploração predatória e clandestina do palmito visivelmente afetou a estrutura ecológica do remanescente florestal estudado, uma vez que houve uma baixa ocorrência de grandes frugívoros e predadores de sementes do fruto da palmeira. O baixo número de registros de frugívoros de grande porte, consequentemente, sugere um quadro de defaunação.

As 16 espécies de aves e mamíferos que consumiram o fruto de $E$. edulis corroboram o papel desta palmeira como espécie-chave na dinâmica florestal. Essa importância é ainda maior pelo fato de apresentar seu pico de frutificação em períodos de escassez ou menor disponibilidade de outros frutos e alimentos, principalmente no inverno.

\section{AgRADECIMENTOS}

Agradecemos à Fepagro, especialmente ao Rodrigo Favreto, pelo suporte e acesso à área de estudo, e também ao Gilson Schlindwein, pelo auxílio e uso da infra-estrutura do Laboratório de sementes da Fepagro de Porto Alegre, e à Universidade La Salle.

\section{REFERÊNCIAS BILBIOGRÁFICAS}

ANDREAZZI, C.S. 2008. Efeitos da fragmentação florestal sobre a fenologia reprodutiva, dispersão e predação de sementes da palmeira Attalea humilis. Dissertação de
Mestrado. Universidade Federal do Rio de Janeiro. 107p.

Andreazzi, C.S.; Pires, A. \& Fernandez, F.A. 2009. Mamíferos e palmeiras neotropicais: interações em paisagens fragmentadas. Oecologia Brasiliensis 13 (4): 554-574.

Ayres, M.; AYres Jr. M.; AYRES, D.L. \& DOS SANTOS, A.S. 2007. BioEstat 5.0 - aplicações estatísticas nas áreas das Ciências Biológicas e Médicas. Disponível em: $<$ http//dv.ict.unesp.br/ivan/downloads/ Bioestat _ $5 *$ Manual-BioEstat_5.pdf>. Acesso em: 2 dez. 2014.

BARRoso, R.M. \& ReIS, A. 2010. Hanazaki, N. Etnoecologia e etnobotânica da palmeira juçara (Euterpe edulis Martius) em comunidades quilombolas do Vale do Ribeira, São Paulo. Acta Botanica Brasilica 24 (2): 518-528.

BECKER, F.G. 2002. Distribuição e abundância de peixes e suas relações com características de hábitat local, bacia de drenagem e posição espacial em riachos de Mata Atlântica (bacia do rio Maquiné, RS, Brasil). Tese de Doutorado. Universidade Federal de São Carlos. 201p.

BORGHETTI, F. \& FERREIRA, A.G. 2004. Interpretação de resultados de germinação. In: FERREIRA, A.G. \& BORGHETTI, F. (eds.). Germinação: do básico ao aplicado. Artmed, Porto Alegre. pp. 209-222.

BREWER, S.W. \& REJMÁNEK, M. 1999. Small rodents 
as significant disperses of tree seeds in a Neotropical forest. Journal of Vegetation Science 10: 165-174.

BUdKE, J.C.; ATHAYde, E.A.; GIEHL, E.L.H.; ZÁCHIA, R.A. \& EISINGER, S.M. 2005. Composição florística e estratégias de dispersão de espécies lenhosas em uma floresta ribeirinha, arroio Passo das Tropas, Santa Maria, RS, Brasil. Iheringia sér. Bot. 60 (1): 17-24.

Cademartori, C.V.; Marques, R.V. \& Pacheco, S.M. 2008. Estratificação vertical no uso do espaço por pequenos mamíferos (Rodentia, Sigmodontinae) em área de Floresta Ombrófila Mista, RS, Brasil. Revista Brasileira de Zoociências 10 (3): 189-196.

CAMpos, R.C.; Steiner, J. \& Zillikens, A. 2012. Bird and mammal frugivores of Euterpe edulis at Santa Catarina island monitored by camera traps. Studies on Neotropical Fauna and Environment 47 (2): 105-110.

CASTRO, E.R.D. 2003. Variação espaço-temporal na fenologia e frugivoria do palmito juçara Euterpe edulis Martius (Arecaceae) em três tipos de floresta atlântica. Dissertação de Mestrado. Universidade Estadual Paulista. 109p.

Coelho de SouzA, G. 2003. Extrativismo em área de Reserva da Biosfera da Mata Atlântica no Rio Grande do Sul: um estudo etnobiológico em Maquiné. Tese de Doutorado. Universidade Federal do Rio Grande do Sul. 197p.

Cordeiro, N.J. \& HowE, H.F. 2003. Forest fragmentation severs mutualism between seed dispersers and an endemic African tree. Proceedings of the National Academy of Sciences 100 (24): 14052-14056.

CÔRTES, M.C. 2006. Variação espacial nas interações entre o palmito Euterpe edulis e as aves frugívoras: implicações para a dispersão de sementes. Dissertação de Mestrado. Universidade Estadual Paulista. 95p.

CULlen JR., L. \& RudRAN, R. 2003. Transectos lineares na estimativa de densidade de mamíferos e aves de médio e grande porte, pp.169-179. In: CULLEN-JR., L. et al. (orgs.). Métodos de estudos em biologia da conservação e manejo da vida silvestre. Curitiba, UFPR e Fundação o Boticário de Proteção à Natureza. 667p.

DALPIAZ, S. 2000. Maquiné e Santo Antônio da Patrulha: elos históricos. In: 1을 ENCONTRO DE PESQUISADORES DO VALE DO RIO MAQUINÉ, Porto Alegre, RS. pp.1-5.

Del-Claro, K. \& Torezan-Silingardi, H.M. 2006. Comportamento animal, interações ecológicas e conservação, pp. 399-410. In: RochA, C.F.D. et al. (eds.). Biologia da Conservação: essências. São Carlos, Rima.: 588p.

FADINI, R.F. 2005. Limitações bióticas afetando 
- recrutamento da palmeira Euterpe edulis em uma ilha continental da Mata Atlântica. Dissertação de Mestrado. Universidade Estadual Paulista. 78p.

FARIAS, M. 2009. Reinventando a relação humano

- Euterpe edulis: do palmito ao açaí. Dissertação de Mestrado. Universidade Federal de Santa Catarina. 85p.

FaVreto, R. 2010. Aspectos etnoecológicos e ecofisiológicos de Euterpe edulis Mart. (Arecaceae). Tese de Doutorado. Universidade Federal do Rio Grande do Sul. $143 p$.

Favreto, R.; Mello, R.S.P. \& De Moura Baptista, L.R. 2010. Growth of Euterpe edulis Mart. (Arecaceae) under forest and agroforestry in southern Brazil. Agroforestry Systems 80 (2): 303-313.

Forget, P.M. 1990. Seed-dispersal of Vouacapoua americana (Caesalpinaceae) by caviomorph rodents in French Guiana. Journal of Tropical Ecology 6 (4): 459-468.

FZB-RS. 2015. Táxons da flora nativa do Estado Rio Grande do Sul ameaçadas de extinção. Disponível em: <http://www.fzb.rs.gov. br/upload/20141208161010 anexo_i_ taxons_da_flora_nativa_do_estado_ rio_grande_do_sul_ameacadas_de_ extincao_1_.pdf>. Acesso em: 05 dez. 2015.

Galetti, M. \& Aleixo, A. 1998. Effects of palm heart harvesting on avian frugivores in the Atlantic rain forest of Brazil. Journal of Applied Ecology 35 (2): 286-293.

Galetti, M. \& Fernandez, J.C. 1998. Palm heart harvesting in the Brazilian Atlantic Forest: changes in industry structure and the illegal trade. Journal of Applied Ecology 35 (2): 294-301.

GAletti, M.; ZipARRO, V.B. \& Morellato, P.C. 1999. Fruiting phenology and frugivory on the palm Euterpe edulis in a lowland Atlantic forest of Brazil. Ecotropica 5: 115-122.

Galetti, M; Pizo, M.A. \& Morellato, P. 2003. Métodos para o estudo da fenologia, frugivoria e dispersão de semente, pp. 422-395. In: CULLEN, Jr.; RUdRAN, R. \& VAlladares-Padua (orgs.). Métodos de estudos em biologia da conservação e manejo da vida silvestre. Curitiba, UFPR. $395 p$.

Galetti, M.; Guevara, R.; Côrtes, M.C.; Fadinı, R.; Von Matter, S.; Leite, A.B.; LABecca, F.; Ribeiro, T.; Carvalho, C.S.; Collevati, R.G.; PIRES, M.M. \& GUIMARÃES JR., P.R. 2013. Functional extinction of birds drives rapid evolutionary changes in seed size. Science 340 (6136): 1086-1090.

Galetti, M.; BovendorP, R.S. \& GuevarA, R. 2015. Defaunation of large mammals leads to an increase in seed predation in the Atlantic forests. Global Ecology and Conservation 3 (3): 824-830.

Guldemond, R. \& VAN AARDE, R. 2007. The 
impact of elephants on plants and their community variables in south africa's maputaland. African Journal of Ecology 45: 327-335.

\section{Henderson, A. 2002. Evolution and Ecology of}

Palms. Bronx, The New York Botanical Garden. 198p.

JoRdANo, P.; GALETTI, M.; PIZO, M.A. \& SilVA, W.R. 2006. Ligando frugivoria e dispersão de sementes à Biologia da Conservação, pp 411-436. In: RochA, C.F.D. et al. (orgs.). Biologia da Conservação: Essências. São Carlos. 588p.

LABECCA, F.M. 2012. Redes de interação entre dispersores de sementes e Euterpe edulis (Arecaceae) em um grandiente de defaunação. Trabalho de Conclusão de Curso. Universidade Estadual Paulista. 47p.

LAPS, R.R. 1996. Frugivoria e dispersão de sementes de palmiteira (Euterpe edulis, Martius Arecaceae) na Mata Atlantica, sul do Estado de São Paulo. Dissertação de Mestrado. Universidade Estadual de Campinas. 319p.

LEMOS, C. A. 2003. Qualidade da água de uma bacia hidrográfica inserida na Reserva da Biosfera da Mata Atlântica, Maquiné, Rio Grande do Sul, Brasil. Dissertação de Mestrado. Universidade Federal do Rio Grande do Sul. 98p.

MANTOVANI, A. 1998. Fenologia e aspectos da biologia floral de uma população de Euterpe edulis Martius na Floresta Atlântica no sul do Brasil. Dissertação de Mestrado. Instituto de Biociências, Universidade Estadual Paulista, Rio Claro. $66 p$.

Marcuzzo, S.; Pagel. S.M. \& Chiappetti, M.I.S. 1998. Reserva da biosfera da Mata Atlantica no Rio Grande do Sul: situação atual, ações e perspectivas. São Paulo, Conselho Nacional da Reserva da Biosfera da Mata Atlântica, Cetesb, v. 11. 48p.

Matos, D.M.S. \& WATKINSON, R. 1998. The fecundity, seed, and seedling ecology of the edible palm Euterpe edulis in southeastern Brazil. Biotropica 30 (4): 595-603.

MIKICH, S.B.A. 2002. Dieta frugívora de Penelope superciliaris (Cracidae) em remanescentes de floresta estacional semidecidual no centro-oeste do Paraná, Brasil e sua relação com Euterpe edulis (Arecaceae). Ararajuba 10 (2): 207-217.

Model N.S. \& SANDER G.R. 1999. Produtividade e característica do fruto de abacaxizeiro em função do preparo do solo de plantio. Pesquisa Agropecuária Gaúcha 5 (2): 209216.

Pinto, S.R.R.; SANTOS, A.M.M. \& TABARElli, M. 2009. Seed predation by rodents and safe sites for large-seeded trees in a fragment of the Brazilian Atlantic forest. Brazilian Journal of Biology 69 (3): 763-771.

PIRES, A. DOS SANTOS. 2006. Perda de diversidade 
de palmeiras em fragmentos de Mata Atlântica: padrões e processos. Tese de Doutorado. Universidade Estadual Paulista. 108p.

PIzo, M.A. \& SIMÃo, I. 2001. Seed deposition patterns and the survival of seeds and seedlings of the palm Euterpe edulis. Acta Oecologica 22 (4): 229-233.

Pizo, M.A.; Silva, W.R.; GaletTI, M. \& LAPS, R. 2002. Frugivory in cotingas of the Atlantic Forest of southeast Brazil. Ararajuba 10 (17): 177-185.

PizO, M.A. \& ViEIRA, E.M. 2004. Palm harvesting affects seed predation of Euterpe edulis, a threatened palm of the Brazilian Atlantic Forest. Brazilian Journal of Biology 64 (3B): 669-676.

Raupp, S.V.; Brack, P. \& de Carvalho leite, S.L. 2009. Aspectos demográficos de palmiteiro (Euterpe edulis Mart.) em uma área da Floresta Atlântica de Encosta, em Maquiné, Rio Grande do Sul. Iheringia Série Botânica 64 (1): 57-61.

REDFORD, K.H. 1985. Foods habits of armadillos (Xenarthra: Dasypodidae), pp. 429-437. In: Montgomery, G.G. (ed.). The Evolution and ecology of sloths, armadillos, and vermilinguas. Washington, Smithsonian Institution. 451p.

REIS, A. 1995. Dispersão de sementes de Euterpe edulis Martius (Palmae) em uma floresta ombrófila densa Montana da Encosta
Atlântica em Blumenau, SC. Tese de Doutorado. Universidade Estadual de Campinas. 154p.

ReIS, A. \& Kageyama, P.Y. 2000. Dispersão de sementes do palmiteiro (Euterpe edulis Martius - Palmae), pp. 60-92. In: REIS, M.S. \& REIS, A. (eds.). Euterpe edulis Martius (palmiteiro): biologia, conservação e manejo. Itajaí, Herbário Barbosa Rodrigues. 335p.

RIBEIRO, T.C. 2012. Efetividade de dispersão de sementes de palmito (Euterpe edulis) em um gradiente de defaunação. Trabalho de Conclusão de Curso. Universidade Estadual Paulista. 46p.

SICK, H. 2001. Ornitologia Brasileira. Rio de Janeiro, Nova Fronteira, 2. ed. 922p.

Silva, M. G. \& TABARELLI, M. 2001. Seed dispersal, plant recruitment and spatial distribution of Bactris acanthocarpa Martius (Arecaceae) in a remnant of Atlantic forest in northeast Brazil. Acta Oecologica 22 (5): 259-268.

TAbarelli, M. \& Mantovani, W. 1999. Regeneration of a neotropical montane forest following slash-and-burn (São PauloBrazil). Revista Brasileira de Biologia 59 (2): 239-250.

Terborgh, J.; Nuñez-Iturri, G.; Pitman, N.C.; Valverde, F.H.C.; Alvarez, P.; SWamy, V. \& PAINE, C.T. 2008. Tree recruitment in an empty forest. Ecology 89 (6): 1757-1768. 
Trolan, L.C. 2009. Contribuições ao manejo sustentável dos frutos de Euterpe Edulis Martius: estrutura populacional, consumo de frutos, variáveis de habitat e conhecimento ecológico. Dissertação de Mestrado. Universidade Federal do Rio Grande do Sul. 86p.

VANDER WALL, S.B. \& LONGLAND, W.S. 2004. Diplochory: are two seed dispersers better than one? Trends in Ecology and Evolution 19: 155-161.

VAN RoOSMALEN, G.M. 1985. Fruits of the Guianan Flora. Netherlands, Institute of Systematic Botany, Utrecht University. 483p.

Vieira, E.; PIzO, M. \& IZAR, P. 2003. Fruit and seed exploitation by small rodents of the Brazilian Atlantic forest. Mammalia 67: 533-540.

WANG, B.C. \& SMITH, T.B. 2002. Closing the seed dispersal loop. Trends in Ecology and Evolution 17 (8): 379-385.

WILLIS, E.O. 1979. The composition of avian com
- Brazil. munities in remanescent woodlots in Southern. Papéis Avulsos de Zoologia 33: 1-25.

WILLIS, E.O. 1989. Aves dispersoras do palmito (Euterpe edulis): a possibilidade de extinção em matas pequenas. In: XVI CONGRESSO BRASILEIRO DE ZOOLOGIA, João Pessoa, Paraíba, Brasil. pp.144.

ZIMMERMANN, C.E. 1990. Nota preliminar sobre a dispersão do palmito, Euterpe edulis, por passeriformes. In: XVII CONGRESSO BRASILEIRO DE ZOOLOGIA, Londrina, Paraná, Brasil. pp.185.

ZIMMERMANN, C.E. 1999. A possível dispersão das sementes de Euterpe edulis (Arecaceae) por aves em ambientes degradados. Revista de Estudos Ambientais 1: 12-17.

Recebido: 28/04/2017

Revisado: 06/06/2017

Aceito: 20/07/2017 